\title{
Histoire et épistémologie de la notion de mise en scène
}

History and epistemology of the notion of "mise en scène"

\section{Roxane Martin}

\section{(2) OpenEdition}

\section{Journals}

Édition électronique

URL : https://journals.openedition.org/pratiques/11254

DOI : 10.4000/pratiques. 11254

ISSN : 2425-2042

Éditeur

Centre de recherche sur les médiations (CREM)

\section{Référence électronique}

Roxane Martin, « Histoire et épistémologie de la notion de mise en scène », Pratiques [En ligne],

191-192 | 2021, mis en ligne le 15 décembre 2021, consulté le 03 janvier 2022. URL : http:// journals.openedition.org/pratiques/11254; DOI : https://doi.org/10.4000/pratiques.11254

Ce document a été généré automatiquement le 3 janvier 2022.

(c) Tous droits réservés 


\title{
Histoire et épistémologie de la notion de mise en scène
}

\author{
History and epistemology of the notion of "mise en scène"
}

\author{
Roxane Martin
}

\section{Introduction}

1 Le festival d'Avignon 2005 a constitué un électrochoc pour les études théâtrales françaises. En désignant J. Fabre comme artiste associé de cette $59^{\mathrm{e}}$ édition, les tout nouvellement nommés directeurs du festival, H. Archambault et V.Baudriller, semblaient vouloir mettre fin au règne du «théâtre de texte » (Py, 2005), ou tout au moins «constituer une lignée artistique singulière» (Talon-Hugon, 2006, p.3), suffisamment fertile pour détrôner le théâtre "dramatique » au profit du théâtre "postdramatique ». Dès lors, la notion de "mise en scène», pierre angulaire des réflexions sur le théâtre depuis plus d'un siècle, ne semblait plus pertinente pour interroger des pratiques performatives que cette $59^{\mathrm{e}}$ édition du festival avait en quelque sorte institutionnalisées. Le théâtre fut alors déclaré «en crise» et les chercheurs s'attachèrent à en identifier les fondements. En 2011, la revue Critique publia un numéro intitulé «La mise en scène: mort ou mutation? ? et P. Roger, dans la préface, pointait le changement de paradigme qui paraissait alors se faire jour sur les scènes contemporaines (p. 835):

Il y a pourtant, dans la crise d'aujourd'hui, quelque chose de nouveau qui signale peut-être un ébranlement en profondeur. Depuis au moins un demi-siècle, le théâtre le plus « moderne », le plus vivant en tout cas, a été dominé par la figure du metteur en scène - flanquée à partir des années 60-70 de celle de "dramaturge ». Or, c'est bien cette figure qui parait aujourd'hui compromise ou dévaluée. C'est toute une manière de faire du théatre et surtout de le «diriger » qui est en cause dans ce qu'on appelle parfois le postdramatique.

2 L'utilisation de l'épithète "moderne » pour qualifier le théâtre qui s'était exprimé majoritairement sur les scènes subventionnées dans la seconde moitié $d u x^{e}$ siècle n'est évidemment pas anodine. Elle convoque ce que J.-P. Sarrazac (2005) a théorisé 
sous la formule "mise en scène moderne " et qui correspond à une conception du théâtre comme " un art à deux temps " (Gouhier, 1989) : celui de l'auteur d'abord, qui conçoit l'œuvre dramatique, celui du metteur en scène ensuite, qui, comme le précise A. Vitez (1995, p. 94), « loin de se contenter de suivre, comme il le croit, les indications de l'auteur, se donne pour tâche de mettre en scène le monde ». Cette autonomie du metteur en scène, conçu comme un artiste à part entière et pouvant à sa guise manier les éléments scéniques dans le but de proposer une interprétation singulière de l'œuvre dramatique (une interprétation souvent actualisée de manière à mettre l'œuvre, même ancienne, en écho avec les problématiques sociopolitiques du monde contemporain), a dominé la création scénique depuis qu'A. Antoine, devenu directeur du Théâtre de l'Odéon en 1906, avait réussi à faire admettre à ses contemporains qu'il était possible de monter les classiques de manières différentes : «comme au temps de Louis XIV » et " dans la tradition comme à la Comédie-Française » (Sarrazac, 1999, p. 21). Remettre en cause la figure centrale du metteur en scène revenait donc à ébranler les fondements théoriques de la notion de « mise en scène » et, par ricochet, à remettre implicitement en cause la légitimité du théâtre en tant qu'art.

3 Une décennie plus tard, après que la notion de "postdramatique " eut été abondamment commentée (et parfois sévèrement critiquée) par les théoriciens, que les artistes eux-mêmes eurent délaissé les formules relativistes et expérientielles pour revenir à la narration, une autre notion s'empara des études théâtrales afin de désigner ce qui apparaissait comme un nouveau paradigme de la création scénique: "les écritures de plateau " (Tackels, 2015). Également sujette aux réticences et aux oppositions, cette notion s'édifiait elle aussi en opposition au "théâtre de mise en scène ». La fabrique du spectacle ne se concevait plus dans la succession de deux moments (l'un textuel, l'autre scénique), mais dans un geste qui s'effectuait depuis le plateau et aboutissait, à l'aide d'un collectif au travail, à une œuvre qui mêlait indistinctement les langages verbal, scénographique, gestuel, sonore, chorégraphique, vidéographique, et pouvait morceler l'espace-temps de la fiction en injectant des éléments performatifs ou des matériaux directement puisés dans le monde « réel ». Ce processus de création, en abolissant la frontière entre les catégories d'auteur et de metteur en scène, s'édifiait donc, à la suite du théâtre postdramatique, comme l'une des formules de la postmodernité.

4 Fondées l'une et l'autre sur l'antagonisme texte/scène, ces deux théories butent sur une aporie que nombre de théoriciens (Benhamou, 2012; Danan, 2013) et d'historiens du théâtre (Biet \& Triau, 2006 ; Lécroart, Peslier \& Piana, 2013) ont cherché à révéler : les premiers en discutant la dichotomie Présentation/Représentation sur laquelle est bâtie la théorie du théâtre postdramatique ; les seconds en dévoilant la présence, dans l'histoire longue des spectacles, de formes pouvant satisfaire les catégories définitionnelles du théâtre postdramatique et des écritures de plateau, invalidant par ce biais leur ancrage dans la postmodernité. Un élargissement de la définition de la «mise en scène » s'avère donc nécessaire afin de lever quelques-unes de ces difficultés. Cette étude entend mettre en perspective les conditions d'émergence de la notion de « mise en scène » dans le paysage théâtral français. Elle montrera que la mise en scène n'engage pas seulement, en amont des expérimentations naturalistes d'A. Antoine, une façon d'organiser les éléments de la représentation. Elle est davantage assimilable à une écriture scénique qui complète le drame, lui accorde une portée sociale, esthétique, politique immédiate. Aussi son analyse invite-t-elle à interroger le rapport entre le 
monde réel et la fiction dramatique. Car la notion de "mise en scène » est bien née d'une volonté de maîtriser la représentation, de gratifier le théâtre d'un rôle social et politique, d'agir sur l'idée qu'un public se fait de son identité culturelle. Le détour par les scènes des $\mathrm{XVIII}^{\mathrm{e}}$ et $\mathrm{XIX}^{\mathrm{e}}$ siècles peut donc s'avérer précieux pour questionner les notions d'authenticité, de fabrication, de vérité dans leur rapport au réel. Il permettra de contextualiser l'avènement de la "mise en scène moderne ", d'identifier le geste inaugural d'A. Antoine dans sa façon d'appréhender nouvellement la relation texte/ scène, de construire les outils théoriques utiles pour interroger les changements de paradigme à l'œuvre dans les créations scéniques d'aujourd'hui.

\section{À l'origine de la « mise en scène » : les dramaturgies du tableau}

5 Il ne s'agit évidemment pas ici de chercher à situer les origines de la mise en scène en tant qu'activité de coordination et d'unification des éléments du spectacle. De tout temps et en tous lieux, le théâtre a eu besoin d'un " coordinateur ", d'une personne ou d'un collectif chargés de régler les éléments de la représentation de manière à lui accorder une unité suffisante pour être rendue intelligible pour le spectateur. Le propos consiste davantage à interroger la mise en scène comme notion, à identifier les raisons historiques, sociales et politiques de son apparition dans la langue. C'est pourquoi nous avons choisi de circonscrire cette étude au contexte français. Une analyse similaire pourrait être effectuée dans d'autres paysages culturels, européens ou extraeuropéens. Le travail colossal qu'une telle enquête suppose, fondé sur le croisement de nombreuses sources, littéraires, politiques, économiques et juridiques, justifie le périmètre géographique qui lui est ici accordé.

6 Dans son célèbre article "Causerie sur la mise en scène », A. Antoine (1903, p. 110) ne s'y était pas trompé :

Le théâtre d'intrigue, le théâtre à situations matérielles apparaît, le théâtre où se marquent la position sociale et la vie journalière des personnages. L'unité de lieu se brise. Figaro saute par les fenêtres et le comte enfonce les portes. Hugo publie la Préface de Cromwell, le grand Dumas se joint à lui : le Moyen Âge chasse l'antiquité : on ne raconte plus, sur la scène, les épisodes tragiques et les combats héroïques: Hernani y ferraille, Saint-Mégrin regarde les astres avant de se rendre chez la duchesse de Guise, et Ruy Blas pousse les meubles devant les portes de sa salle basse pour mourir en paix. Géronte, Célimène et Sganarelle font place à Marguerite Gautier, à Giboyer, au père Poirier ; on mange sur le théâtre, on y dort, on s'assied sur son lit pour rêver comme Chatterton. La mise en scène vient de naître, et, docile, elle va désormais suivre la production dramatique.

7 En associant la naissance de la mise en scène à l'apparition d'un théâtre de l'image, qui se met en place en même temps qu'il se théorise dans la seconde moitié du XvIII siècle, A. Antoine construit la ligne qui relie D. Diderot à É. Zola et inscrit le naturalisme théâtral dans le sillage des dramaturgies du tableau' ${ }^{1}$. Cet examen paraît d'autant plus intéressant qu'il assimile l'émergence de la mise en scène à une réforme de l'écriture dramatique. La matière scénique, revendiquée comme une composante essentielle du drame par les philosophes des Lumières, a pénétré l'écriture théâtrale au point de faire voler en éclat l'ancien système des genres. La loi Le Chapelier du 13 janvier 1791, en autorisant « tout citoyen à élever un théâtre public, et y faire représenter des pièces de tous les genres» (art. 1), a joué un rôle déterminant pour promouvoir ce type de 
dramaturgie. Jusqu'alors contraints par les monopoles des théâtres royaux, les auteurs purent expérimenter plus librement une écriture composite, mêlant des éléments jusque-là appréciés comme disparates parce qu'ils relevaient de modes d'expression appartenant à des genres distincts, distribués entre les théâtres selon le système des privilèges dramatiques ${ }^{2}$. Le geste, le mot, la musique et l'image se sont alors accordés dans d'habiles combinatoires, donnant corps à une écriture scénique qu'on allait bientôt nommer « mise en scène ».

8 Si le « drame bourgeois » théorisé par D. Diderot a largement contribué à déplacer le centre de gravité de l'écriture théâtrale en faisant en sorte que le support de l'action dramatique ne soit plus le discours, mais bien l'image (le tableau) à laquelle l'ensemble des éléments scéniques (musique, corps, décors, mimique, discours et voix) concourent à donner forme, il faut attendre la Révolution pour que le terme " mise en scène » se banalise. Cette observation est essentielle pour l'historien qui cherche à identifier les facteurs ayant favorisé la création d'un terme neuf pour caractériser une activité somme toute assez courante. Si l'on évoque volontiers la «mise » d'une pièce sous l'Ancien Régime (Razgonnikoff, 2006), si l'on trouve déjà l'expression " mise en scène " dans sa forme substantivée dans quelques encyclopédies spécialisées à l'orée des années $1790^{3}$, le terme rejoint le langage usuel dans les années 1795-1800, sous l'impulsion des politiques. Car ce que propose le théatre pendant la décennie révolutionnaire, c'est une rupture dans les modalités mimétiques. L'on retrouve, pour simplifier, deux types de spectacles sous la Révolution. Le premier rejoint ce que P. Frantz (1992, p. 522) a nommé le « théâtre de l'urgence » qui, puisant dans les savoirfaire développés sur les théâtres de la Foire sous l'Ancien Régime, fait « cause commune avec la Révolution par la transposition sur scène, non plus sporadique mais presque systématique, d'un fait tiré de l'actualité " (Tissier, 1992, p. 32). Ces pièces de circonstance, composées en quelques jours, proposent une intrigue rudimentaire, ponctuées de combats, danses, airs chantés et changements de décor qui formaient les ingrédients obligés des pantomimes de la Foire. Ces éléments sont désormais construits en lien direct avec l'actualité. La fiction dramatique, portée par une intrigue souvent fondée sur le thème des amours contrariées, se voit ainsi rompue par des chants républicains, des danses autour des symboles de la République (l'arbre de la liberté), des évolutions militaires évoquant les combats de l'armée révolutionnaire, des lectures de comptes-rendus des débats menés conjointement à l'Assemblée, des apothéoses dramatiques célébrant les martyrs de la Révolution (J.-P. Marat, N. Beaurepaire). L'on comprend, dans cette perspective, les contestations qu'ont pu émettre les historiens du théâtre à l'égard des théories du postdramatique ou des écritures de plateau. La représentation ne repose pas, dans ce cas de figure, sur une valorisation de la fiction dramatique, mais cherche à travers elle à traduire l'événement en symbole, c'est-à-dire à susciter l'adhésion des publics en favorisant leur inscription dans l'Histoire en train de s'écrire, autrement dit dans le temps de la Révolution.

9 L'autre type de spectacle proposé sur les scènes de la décennie révolutionnaire repose sur des œuvres dramatiques anciennes, dont la représentation était désormais permise sur tous les théâtres. Dans son rapport prononcé le 13 janvier 1791 à l'Assemblée constituante, I. Le Chapelier l'avait clairement précisé : «Le public devait avoir la propriété de ces chefs-d'œuvre; [...] chacun devait être maître de s'emparer des ouvrages immortels de Molière, de Corneille et de Racine, pour essayer d'en rendre les beautés et de les faire connaître. » La représentation théâtrale allait jouer un rôle dans 
l'actualisation de ces chefs-d'œuvre. Il s'agissait de moderniser, par le biais de la scène, un répertoire devenu patrimoine, c'est-à-dire chose du peuple. Et pour que ce peuple puisse s'approprier cet ensemble d'œuvres dont le régime monarchique l'avait privé, il fallait l'inscrire dans le temps présent, lui accorder une historicité dans laquelle tous les citoyens pouvaient prendre place. En d'autres termes, il fallait faire participer le public en révélant le caractère actuel de ces pièces quitte à leur faire dire autre chose que ce qu'elles disaient en leur temps. En bref, il s'agissait de faire entrer le répertoire "classique» dans l'Histoire. Ce répertoire, au préalable propriété exclusive de la Comédie-Française, fut ainsi soumis à une réécriture tant scénique que dialogique de manière à satisfaire l'idéal révolutionnaire (Poirson, 2008). L'on comprend dans cette perspective toute l'importance qu'allait revendiquer la notion de « mise en scène ». Elle devait se concevoir, non pas comme une opération d'ornementation d'un texte dramatique, mais comme une "réécriture », une «refonte » de l'œuvre de manière à l'inscrire dans le temps présent du collectif-nation.

Devenu en partie l'organe d'un État qui, dès 1791, invente et instaure la pratique de la politique culturelle à des fins d'autolégitimation, d'identité nationale et d'instruction publique, le théâtre s'est rapidement édifié comme le lieu et l'enjeu de tous les affrontements. Aussi les dirigeants révolutionnaires ont-ils vite compris qu'il fallait exercer un contrôle attentif sur les spectacles, et mesurer, en amont de la représentation, les connotations qui pouvaient être véhiculées par la scène exclusivement. C'est là que l'on voit apparaître, notamment après la chute du régime de Robespierre, des directives précises adressées aux censeurs. Ceux-ci eurent pour tâche d'exiger, au sein des pièces qui étaient soumises à leur examen, une description précise des éléments du spectacle. Prenant la forme de longues didascalies, cette description venait compléter les dialogues en précisant le moindre détail de la représentation : depuis la couleur des costumes (l'usage du blanc, par exemple, pouvait être perçu comme un ralliement aux royalistes) jusqu'à l'accessoire utilisé comme support à une pantomime (une simple estrade pouvait évoquer l'échafaud sur lequel avaient péri tant de citoyens sous la Terreur). Cette politique culturelle ne fut pas étrangère à l'émergence d'une "écriture du spectacle» que l'on finit par nommer «mise en scène ». L'analyse des rapports produits par la police des spectacles entre 1795 et 1799 le confirme. Les censeurs utilisent d'abord les verbes "mettre en scène " ou «mettre sur la scène » à propos d'un personnage, d'une pièce ou d'un sujet. Puis progressivement la formule «mise en scène » apparaît dans sa forme substantivée pour qualifier tout ce qui appartient au registre du spectaculaire. Cet exercice imposé aux auteurs et directeurs de théâtre a sans aucun doute favorisé la banalisation du terme qui, à partir de 1800, fait pleinement partie du vocabulaire dramatique. On le retrouve alors partout mentionné : sur les brochures des pièces qui précisent bien souvent le nom de «l'auteur de la mise en scène » (qui n'est pas nécessairement le même que celui des dialogues), dans les rapports administratifs produits par les différents services de l'État, dans les critiques dramatiques publiées dans la presse ${ }^{4}$.

11 La notion de «mise en scène » est donc apparue dans le contexte culturel français par le truchement de deux facteurs. Elle s'inscrit d'abord en lien direct avec la réforme de l'écriture dramatique proposée par les philosophes des Lumières (c'est l'argument défendu par A. Antoine), qui reposait sur une conception de la représentation théâtrale comme totalité sémantique. La notation de la pantomime, la description scénographique répondaient à une volonté de l'auteur de s'approprier la scène afin d'élaborer une dramaturgie qui, pour faire sens, devait incorporer les différentes 
formes de langages scéniques. Mais le contexte révolutionnaire a aussi créé les conditions pour que soit envisagé autrement le rapport entre le monde réel et la fiction dramatique. Convoqué au sein de la fable, l'événement politique devenait symbole, c'est-à-dire fixé, grâce à la médiation de la scène, dans la mémoire collective. On assiste alors à une inversion du processus d'écriture. Le texte dramatique n'est pas l'élément premier à partir duquel s'élabore la représentation; simple matériau, il se modèle en fonction des impératifs d'un art théâtral qui accorde à la scène un pouvoir social et politique. À l'époque de son émergence, la notion de "mise en scène » ne se définit donc pas dans un rapport texte/scène. Elle signale plutôt l'apparition d'une "autre voix ", celle qui consiste à dire quelque chose du monde présent dans l'ici et le maintenant de la représentation.

\section{La mise en scène comme outil de conquête des scènes subventionnées}

12 Le rétablissement du système des privilèges dramatiques, décrété par Napoléon en 1806-07, a considérablement freiné l'essor d'une mise en scène conçue comme langage scénique autonome, c'est-à-dire pouvant être élaboré indépendamment des conventions d'écriture propres aux genres auxquels les théâtres étaient affectés par privilège. Sur le modèle de l'Ancien Régime, les théâtres de l'État récupèrent leur répertoire et leurs privilèges anciens. Les théâtres privés, désormais nommés « théâtres secondaires ", sont limités à quatre à Paris, à un dans les grandes villes de province. Leur répertoire est restreint aux genres du vaudeville et du mélodrame exclusivement.

13 Héritier des dramaturgies du tableau, le mélodrame est le seul genre autorisé, après 1807, à pratiquer une forme de "mélange des genres ", c'est-à-dire une écriture qui associe étroitement le verbe, le geste, la musique et l'image. Les éléments scéniques, consubstantiels de sa dramaturgie, sont distribués dans la structure d'ensemble de manière à conduire le pathétique à son point d'extrême violence. Autant dire que le mélodrame exige une maîtrise avisée de la scène. C'est sans doute pourquoi la notion de "mise en scène" se voit presque exclusivement attachée à ce genre jusqu'aux dernières années de la Restauration. Des régisseurs «chargés de la mise en scène des mélodrames » apparaissent même dans les registres du personnel des théâtres du mélodrame à partir de 1820 .

14 Cette étude n'est pas le lieu pour approfondir en détail les procédés d'écriture du mélodrame ${ }^{5}$. Précisons toutefois qu'au cours des années 1800-1820 le mélodrame a mis sur pied une esthétique scénique qui, à partir des années 1825-1830, s'est vue appliquée à tous les genres dramatiques. C'est d'ailleurs au sein d'un théâtre de mélodrame, le Panorama-Dramatique, que s'est formée l'équipe artistique appelée à réformer les pratiques scéniques de la Comédie-Française en 1825. À cette date, l'ancien directeur du Panorama-Dramatique, le baron Taylor, est nommé administrateur du premier théâtre français. Il convoque son ancienne équipe de costumiers et de décorateurs, et surtout il crée un poste de "directeur de la scène " pour celui qui avait occupé les mêmes fonctions au Panorama-Dramatique: J.-L. Solomé. Immédiatement, la fonction de " directeur de la scène » (autrement nommé «metteur en scène ») est créée dans les autres théâtres royaux. J.-L. Solomé passe à l'Opéra en $1828 ; \mathrm{H}$. Albertin (un auteur de mélodrame) prend sa place à la Comédie-Française; R.-Ch. Guilbert de Pixerécourt (reconnu comme le "père du mélodrame ») est nommé à la tête de l'Opéra-Comique en 
1824 et met en scène l'ensemble des œuvres représentées sur ce théâtre pendant les trois années de sa direction. Une pratique de la mise en scène, initiée avec le mélodrame, s'est donc mise en place dans tous les théâtres académiques. Elle a constitué un outil essentiel dans la réforme dramatique portée par les romantiques.

La modernisation du répertoire n'entre pas dans les priorités du baron Taylor lorsqu'il prend ses fonctions à la Comédie-Française en 1825. Désireux avant tout d'imposer sur le premier théâtre français une esthétique scénique nouvelle et de provoquer, par son biais, le délitement de la hiérarchie des genres instituée par l'académisme, le baron Taylor concentre l'essentiel de ses efforts de mise en scène sur les pièces contemporaines. Il déploie alors un budget considérable pour la représentation d'œuvres habilement choisies de façon qu'elles couvrent le panel des genres faisant partie du privilège de la Comédie-Française : la tragédie avec Léonidas de M. Pichat (qui avait été son collaborateur au Panorama-Dramatique); la comédie avec Les Trois Quartiers de L.-B. Picard et Éd. Mazères ; le drame avec Henri III et sa cour d'A. Dumas. Les livrets de mise en scène de ces deux dernières pièces furent d'ailleurs publiés ${ }^{6}$, ce qui montre combien le baron Taylor avait édifié un programme de déstabilisation des usages traditionnels de la Comédie-Française, dont la mise en scène constituait l'épicentre. Après que ces dramaturgies nouvelles se furent imposées dans l'antre de Melpomène, l'idée vint tout naturellement de "renouveler " la mise en scène des œuvres du répertoire afin de les mettre en accord avec les enjeux du monde contemporain. E. Laugier (1844, p.60), alors archiviste de la Comédie-Française, le confirme:

Les hommes de l'avenir, la partie agissante de notre littérature, se donnaient rendez-vous à la représentation des œuvres de MM. Alfred de Vigny, Victor Hugo et Casimir Delavigne. Grâce aux bénéfices réalisés par les nouveautés en faveur, il devenait possible d'apporter un soin minutieux à l'exécution et à la mise en scène des œuvres classiques; les salons de Molière étaient devenus propres et élégants ; parée de nouveaux atours, l'ancienne comédie avait repris un air de jeunesse qui lui allait à ravir. Au théâtre, les détails matériels ont de grandes conséquences morales, et nous avouons, pour notre part, que nous aimons l'appartement de Célimène richement meublé, rempli des visiteurs de l'adorable coquette, eux étalant tout le luxe des habits de l'époque, elle parée comme une reine qu'elle était; cette magnificence des vêtements nous rappelle le grand siècle, le vers si naturel et si parfait de Molière nous semble plus naturel et plus parfait encore, et nous en saisissons mieux toutes les intentions.

Cette volonté de représenter le répertoire « classique » avec le mobilier et les costumes de son temps (c'est-à-dire tels qu'on se les représente à l'époque) est une idée qui a innervé la réflexion sur l'art de la mise en scène jusqu'à au moins A. Antoine. C'est pourquoi il faut comprendre la mise en scène comme un discours historiographique, susceptible de reconstruire le passé à l'aune des enjeux sociopolitiques du présent. Représenter Molière avec le mobilier et les costumes de son époque, c'est bien sûr substituer de neuves traditions scéniques à des anciennes, mais c'est aussi interroger les productions du «Grand Siècle » et les mettre à la portée du regard contemporain. Selon une démarche similaire à celle entreprise sous la Révolution, la mise en scène s'impose comme un langage; il ne s'agit plus ici d'orienter la lecture des œuvres de manière à satisfaire les idéaux de la Révolution (et par ce moyen à éduquer le sens civique du spectateur), mais de construire, par le théâtre, une histoire au sein de laquelle le «Grand Siècle » ne serait plus l'épicentre, mais une simple parenthèse dans l'histoire longue du peuple français. 
17 Cette démarche est particulièrement prégnante dans l'Antigone de Sophocle, adaptée et mise en scène par A. Vacquerie et P. Meurice en 1844 au Théâtre de l'Odéon. L'ambition des deux dramaturges, futurs exécuteurs testamentaires de V. Hugo, est clairement formulée dans la préface de l'œuvre, éditée dans une version conforme à celle qui fut représentée (Vacquerie \& Meurice, 1844, p. 7) :

On peut s'assurer maintenant si la révolution littéraire d'il y a quinze ans n'a été qu'une invasion de barbares sans passé, un tremblement de terre accidentel après lequel chacun redresse sa maison, une émotion produite par des moyens transitoires qui devait passer avec ses moyens. On peut reconnaître si c'est le drame qui n'est qu'un art de rencontre sans racines dans le sol. L'œil le moins ouvert peut voir si c'est la tragédie qui descend des tragiques grecs, et si l'étiquette fait le vin. Le drame et la tragédie sont une bonne fois en présence:- d'un côté, Eschyle, Sophocle, Euripide, Shakespeare, Calderón, Goethe, Schiller, Hugo ; - de l'autre, Racine.

Prenant appui sur une autre mise en scène d'Antigone, proposée par K. Stawinsky au Schauspielhaus de Berlin en 1842 et conçue à partir de la partition d'orchestre de F. Mendelssohn-Bartholdy ${ }^{7}$, A. Vacquerie et P. Meurice cherchaient donc à montrer qu'il existait une continuité entre la tragédie grecque et le drame romantique, que celle-ci reposait sur une écriture mêlant les éléments verbaux et scéniques. Présentée dans la préface comme une reconstitution du spectacle tel qu'il s'était donné en Grèce antique, l'Antigone de A.Vacquerie et P. Meurice n'a évidemment plus grand-chose à voir avec son modèle sophocléen. Ce sont bien les codes scéniques du romantisme que l'on retrouve convoqués dans cette mise en scène. Mais l'on comprend que ce spectacle permet de réactiver un romantisme théâtral que la critique semblait avoir trop rapidement enterré. Extirpé du cadre étroit des batailles littéraires qui l'avaient réduit à un art de l'antithèse, il se confondait désormais au mouvement qui rassemblait dans un même souffle toutes les expressions dramatiques européennes depuis l'antiquité, hormis celles qui avaient abouti à la dramaturgie classique. Dans cette perspective, ce n'était plus le romantisme qui était une parenthèse dans l'histoire du théâtre français, mais bien le classicisme qui constituait une rupture et un écart dans l'histoire littéraire européenne.

Cette relecture de l'histoire du théâtre par le biais de la mise en scène ne fut pas circonscrite au seul exemple de la tragédie grecque. Des historiens s'engagèrent dans cette voie, de façon à convaincre les contemporains que les techniques modernes de la mise en scène offraient les outils méthodologiques permettant de reconstruire le passé selon une chaîne ininterrompue, depuis les Grecs jusqu'au romantisme. É. Morice, par exemple, publia en 1835 plusieurs articles dans la Revue de Paris consacrés à « La mise en scène depuis les mystères jusqu'au Cid». D'autres allèrent dans le même sens, comme Ch. Magnin qui publia un article intitulé « De la mise en scène chez les anciens " dans la Revue des deux mondes en 1840, ou encore P. Paris qui bâtit son cours du Collège de France en 1855 sur le sujet : De la mise en scène des mystères et du mystère de la Passion. Chaque fois il s'agissait de convoquer les ingrédients des scènes contemporaines afin de montrer leur syncrétisme comme une invention déjà portée en germe par les scènes antérieures au classicisme. La thèse que défend P. Paris est clairement formulée : le détour par les formes du passé permet de montrer que l'histoire a eu recours à « d'autre mise en scène que [celle en usage] pour la tragédie de Racine » (Paris, 1855, p.5). L'enjeu de ces auteurs n'était donc pas de restituer les conditions sociales et politiques qui avaient façonné les œuvres et pouvaient expliquer leurs représentations et leur réception dans leur contexte historique respectif; il s'agissait de promouvoir 
l'autonomisation d'un art de la mise en scène qui n'était plus assujetti aux conventions dramaturgiques des différents genres dramatiques, mais qui tendait au contraire à les rassembler sous une esthétique commune, celle précisément que le romantisme, par la peinture scénique, le jeu de l'acteur, la chorégraphie et la musique, avait mise sur pied.

La mise en scène appliquée aux œuvres du passé devient donc le grand sujet qui innerve les débats sur l'art théâtral depuis les années 1840 jusqu'à la fin du xix siècle. Des critiques, tel T. Gautier dans La Presse du 18 janvier 1847, défend ce principe :

Molière, sous sa vraie physionomie, est, pour ainsi dire, inconnu au théâtre, et la Comédie-Française accomplirait un devoir pieux en le représentant avec les accessoires qu'il a lui-même jugés nécessaires au succès de ses pièces. Qu'on ressuscite ainsi toutes ses comédies-ballets, en y joignant le chant, la danse, les décorations, les costumes, et l'on aura un spectacle du plus vif attrait et de la plus grande nouveauté. [...] De même il faudrait, ce nous semble, jouer les tragédies en costumes d'époque, avec des casques à panaches, des tonnelets et des perruques infolio.

21 D'autres critiques, en revanche, s'y opposent avec fermeté. Dans le Journal des débats du 21 janvier 1850, J. Janin écrit à propos de L'Amour médecin repris à la ComédieFrançaise :

«L'Amour médecin, disait l'affiche, arrangé par un metteur en scène » et ce metteur en scène n'était rien moins que M. Alexandre Dumas, qui voulut bien, pour cette fois seulement, venir en aide à l'esprit de Molière. Cette mise en scène ne fut pas heureuse ; - M. Alexandre Dumas avait imaginé d'encadrer l'intermède de Molière dans un intermède de la composition de l'auteur d'Antony, et il arriva, chose étrange et chose incroyable, et toute à la louange du poète moderne, que cet habile et intelligent auditoire du Théâtre-Français confondit d'un bout à l'autre, de ces trois petits actes, beaucoup trop allongés, le principal et l'accessoire, la comédie et la mise en scène, la sauce et le poisson.

Confondre le principal et l'accessoire, c'est-à-dire, pour reprendre la métaphore de J. Janin, perdre le goût du poisson sous la sauce, voici finalement le reproche que la critique conservatrice allait formuler à l'encontre de toutes les tentatives menées pour «moderniser» les traditions scéniques de la Comédie-Française. Car il faut bien comprendre les enjeux du débat. "Moderniser", aux yeux de ceux qui défendent l'usage de la mise en scène dans la représentation des œuvres classiques, c'est «restituer » l'œuvre dans son contexte de création, lui redonner les composantes spectaculaires qu'elle avait prétendument perdues au contact des Comédiens-Français, et par ce biais révéler une continuité esthétique entre les œuvres théâtrales du Grand Siècle et le drame contemporain. C'est une manière de se réapproprier les "chefs d'œuvre » du passé - circonscrits par l'absolutisme monarchique au seul plaisir du public aristocratique - de manière à réinscrire leur interprétation dans l'Histoire et fonder le socle de la culture française sur une conception élargie de la nation. Cette ambition pose un problème aux détracteurs de la mise en scène. Il est explicitement formulé par J. Janin qui, dans le Journal des débats du 10 avril 1848, à propos d'une mise en scène d'Horace de Corneille dans laquelle le texte avait été amputé de manière à coller aux événements politiques de 1848, écrit :

C'est ridicule, et c'est odieux; ce serait le plus grand attentat qui se pût commettre contre une nation que d'attenter à ses chefs-d'œuvre, que de se méfier à ce point de sa justice, de son bon sens! Eh! c'est déjà trop, beaucoup trop d'avoir effacé du frontispice de nos collèges les noms glorieux de Henri IV et de Louis-le-Grand! Estce qu'on touche à l'Histoire? [...] Brisez des marbres, arrachez des statues de leur piédestal, déchirez des estampes, à la bonne heure, mais rappelez-vous que la 
parole d'un homme de génie est impérissable, et surtout la parole des poètes;

l'Évangile l'a dit : «Ce qui est écrit est écrit !» littéraire de la France, autrement dit fragiliser les fondations d'un patrimoine sur lequel devait se construire l'identité nationale. La "mise en scène» des classiques engage donc une manière de gérer l'héritage du Grand Siècle et le passé monarchique de la France. Dans la continuité de J. Janin, F. Sarcey formulera des reproches similaires à É. Perrin, alors administrateur de la Comédie-Française, lorsqu'il entreprendra dans les années 1880 de monter les œuvres de Racine avec les outils de la mise en scène naturaliste. Perçue comme la partie "accessoire » du théâtre, la mise en scène était dénoncée comme un matérialisme écrasant (travestissant) l'idée du poète. L'antagonisme "immatériel/matériel », c'est-à-dire l'idée formulée par le poète et sa concrétisation en images par le metteur en scène, s'invitait au cœur de la réflexion sur l'art dramatique. Elle forme le contexte théorique dans lequel s'épanouit la réforme portée par A. Antoine : au Théâtre-Libre d'abord, puis sur la scène de l'odéon à partir de 1906.

\section{Vers la reconnaissance d'une esthétique scénique plurielle}

Nous ne reviendrons pas ici sur le naturalisme d'A. Antoine, finement analysé par J.P. Sarrazac (1999). Précisons simplement que le geste fort d'A. Antoine, par le biais duquel il a su se démarquer des autres metteurs en scène naturalistes de son époque (A. Lemoine-Montigny, É. Perrin, P. Porel), repose sur l'inversion du processus de création scénique. Tandis que P. Porel, par exemple, mentionné par Antoine dans sa «Causerie », dirige ses comédiens sur un plateau nu, A. Antoine exige en premier lieu la constitution du «milieu », c'est-à-dire du décor dans lequel se développe l'action, qu'il aura construit au préalable, sur le papier, à partir d'une analyse du texte représenté. Cette méthode lui permet d'échapper aux traditionnelles plantations de décors encore en usage sur les scènes romantiques. Il l'écrit dans sa "Causerie sur la mise en scène » (Antoine, 1903, p. 113-114) :

Sentez-vous combien, ce premier travail effectué, il deviendra commode et intéressant, après avoir examiné ce paysage ou cet appartement sous toutes ses faces, de choisir le point exact où devra se faire la section qui nous permettra d'enlever le fameux quatrième mur, en maintenant au décor son aspect le plus caractéristique et le plus adéquat à l'action? [...]

Vous comprenez quelle importance prennent peu à peu ces considérations lorsqu'il s'agit de planter un décor. Les Allemands et les Anglais n'hésitent pas: ils combinent, ils coupent, ils biaisent ingénieusement, de façon à ne présenter dans la partie centrale du tableau que la cheminée, la fenêtre, le bureau, le coin d'intérieur dont ils ont besoin.

Ces plantations si pittoresques, si vivantes, pleines d'imprévu et d'intimité, sont trop négligées en France parce que nos metteurs en scène restent influencés malgré tout par le souvenir de nos éternelles dispositions classiques. Un manque de symétrie leur paraîtrait insoutenable à l'œil.

Ce renouvellement de la plantation des décors, qui privilégie en effet la dissymétrie ${ }^{8}$, lui permet d'utiliser le milieu (la partie matérielle de la mise en scène) pour générer l'interprétation de l'œuvre (la partie immatérielle de la mise en scène). Diriger un comédien sur un plateau nu, c'était nécessairement construire l'interprétation par le 
biais du seul texte dramatique. A. Antoine place ses comédiens sur une scène déjà chargée de ses décors dans le but de permettre au «milieu » d'imprégner le jeu et les déplacements des comédiens'. L'on comprend mieux, dans cette perspective, la revendication du lien entre son naturalisme et les dramaturgies du tableau. Le contenu sémantique de l'œuvre dramatique s'élabore bien à partir de l'image scénique (le « tableau », spécifiquement nommé dans le texte cité ci-dessus).

Ce processus de mise en scène aura certainement aidé A. Antoine dans sa tentative réussie de "moderniser » les classiques. L'autre élément, qui nous semble fondamental pour comprendre la totalité de la réforme portée par A. Antoine, est ainsi exprimé dans «Causerie sur la mise en scène » (Antoine, 1903, p. 120):

J'aurais voulu dire toute mon admiration pour le théâtre classique et l'étonnement que j'éprouve, à voir qu'on examine gravement la possibilité d'en renouveler, d'en moderniser la mise en scène. Je voudrais, au contraire, si j'avais un jour l'honneur de diriger un théâtre d'État, revenir en arrière et restituer à nos chefs-d'œuvre le cadre vrai qui leur convient, celui de leur temps. Je voudrais Racine représenté avec les habits de cour de l'époque, dans des décors simples et harmonieux, sans l'appareil extérieur qui puisse atténuer l'effet de son génie.

Par ces quelques lignes, A. Antoine s'inscrit dans la continuité des propos tenus par les romantiques dans les années 1840. Car il faut bien situer le contexte qui motive l'écriture de cet article en 1903. A. Antoine visait de longue date la direction d'un théâtre d'État. Il avait certes partagé la direction du Théâtre de l'Odéon avec P. Ginisty en 1896, mais la politique artistique qu'il chercha à mettre en œuvre suscita le désaveu de son collaborateur qui, de concert avec les autorités ministérielles et quelques mois seulement après avoir éprouvé cette association infructueuse, démissionna afin de pouvoir être renommé seul directeur. Après cette démission forcée, A. Antoine s'apprêtait donc à revenir en vainqueur. En 1903, il publiait «Causerie sur la mise en scène » dans laquelle il exprimait clairement sa volonté de diriger un théâtre d'État; en 1904, il montait Le Roi Lear de Shakespeare au Théâtre-Antoine, qui lui ouvrit effectivement les portes de l'Odéon en 1906. La "modernisation" du répertoire classique par la mise en scène pouvait donc être opérée.

Pour bien saisir le sens des propos d'Antoine, il convient de lire la suite qu'il donne aux quelques lignes citées plus haut (Antoine, 1903, p. 120) :

Toute recherche de couleur locale ou de vérité historique me parait vaine pour de pareils chefs-d'œuvre : aux yeux d'un contemporain de Périclès, Lekain ou Talma auraient semblé aussi peu grecs que Baron. Je crois fermement que c'est altérer la signification de ces merveilleuses tragédies que de les « situer », sinon dans le pays et dans le temps où elles sont nées.

Baron (1653-1729), comédien de la troupe de l'Hôtel de Bourgogne, avait été le créateur des premiers rôles raciniens. Lekain (1729-1778) et Talma (1763-1826), sociétaires de la Comédie-Française, étaient réputés pour avoir, les premiers, uniformisé le costume au personnage en revêtant la toge antique. En les plaçant côte à côte, A. Antoine veut montrer qu'il n'existe pas plus d'authenticité dans le jeu de Lekain et Talma que dans celui de Baron (vêtu selon les conventions du XVII ${ }^{\mathrm{e}}$ siècle, à savoir en costume de cour). La "couleur locale», qui avait été le principe fondateur de la réforme scénique entamée par les philosophes de Lumières, n'était donc qu'une convention scénique parmi d'autres. Aussi A. Antoine rejoint-il l'idéologie défendue par les historiens du théâtre dans la seconde moitié du XIX ${ }^{e}$ siècle : «restituer » les chefs-d'œuvre du passé ne revient pas à adapter le décor et le costume au lieu et à l'époque de la fiction, mais 
bien à les replacer dans le lieu et l'époque de leur création. Tandis que ses prédécesseurs s'étaient heurtés à une critique virulente, défendant l'idée que la mise en scène était inhérente à l'œuvre dramatique et que la Comédie-Française, par son ancrage dans l'Ancien Régime, était seule garante des justes traditions scéniques pour les représenter, A. Antoine allait parvenir à concrétiser leur ambition et révéler la mise en scène comme une esthétique scénique plurielle.

De 1906 à 1913, il monte donc plusieurs de ces «chefs-d'œuvre » du patrimoine en prenant soin de multiplier les esthétiques scéniques. Pour Andromaque (1912) par exemple, il propose deux mises en scène, l'une reconstituée comme au temps de Louis XIV, l'autre jouée dans les traditions de décors et de costumes en vigueur au ThéâtreFrançais. Ce moyen judicieux permettait de rendre caduque la prétendue « authenticité » dont se réclamaient les Comédiens-Français. Il réitère une expérience similaire avec Le Cid (1907), monté dans une mise en scène voulue conforme à celle donnée au Théâtre du Marais en 1636: "Même rampe composée de chandelles de cire, mêmes gentilshommes qui encombrent la salle sans troubler le déroulement de l'action, mêmes costumes somptueux dans un décor Louis XIV »(Sanders, 1978, p. 149). Pour Britannicus (1911), A. Antoine fait jouer les personnages de Néron et de Britannicus par des actrices (Marie Ventura et Andrée Pascal), ce qui lui permet d'introduire un jeu de commentaire entre une tradition théâtrale (le travestissement) et les codes de réception du public contemporain. Le renouvellement de la mise en scène des œuvres $\mathrm{du}$ répertoire dramatique national, en chantier depuis la décennie révolutionnaire, était cette fois-ci durablement entériné.

\section{Le « théâtre de mise en scène » : une forme historique?}

31 Au terme de cette présentation des enjeux théoriques qui se sont greffés autour de la notion de "mise en scène » sur un long XIX $x^{e}$ siècle, il convient de se demander si le "théâtre de mise en scène » est une forme parfaitement datée, qui a aujourd'hui terminé son histoire. Si les théories du théâtre postdramatique et des écritures de plateau ont pu revendiquer leur démarcation avec lui, c'est précisément parce que la notion de " mise en scène » a toujours été définie à travers le couple texte/scène. Cette lecture se justifie au regard de l'historiographie théatrale de la seconde moitié du $\mathrm{xx}^{\mathrm{e}}$ siècle qui a valorisé la figure d'A. Antoine en lui attribuant l'invention de la mise en scène moderne parce qu'il aurait le premier affirmé la dimension herméneutique du travail scénique. Comme le précise A. Folco (2013, p. 49) :

On peut faire l'hypothèse que cette mise en valeur dans les histoires du théâtre correspond à une double évolution du champ institutionnel : au moment où les pouvoirs publics donnent aux metteurs en scène un rôle majeur dans la mise en place du théâtre public, et où les études théâtrales se développent de manière spécifique à l'université comme au CNRS, une autre manière de raconter le fait théâtral se met progressivement en place. Cette nouvelle histoire élabore sa généalogie et sa chronologie propres, et une nouvelle série de noms et d'événements pertinents complète, puis se substitue, à celle que l'histoire littéraire avait établie. C'est dans ce contexte d'élaboration d'une nouvelle mémoire, à la fois artistique, professionnelle et savante, que l'on doit probablement intégrer la valorisation d'Antoine. 
Une analyse attentive des enjeux qui ont innervé la réflexion sur la mise en scène tout au long du XIX siècle montre pourtant que celle-ci ne se définit pas seulement comme une lecture singulière d'un texte dramatique. L'apparition du terme dans la langue s'opère dans un contexte politique particulier, où s'exprime le besoin de gratifier le théâtre d'un rôle social, politique, pédagogique. Que la mise en scène s'épanouisse au sein des dramaturgies du tableau, par le biais desquelles les auteurs cherchent par le maniement des éléments scéniques à véhiculer du sens et des valeurs susceptibles de réformer la société contemporaine, ou bien qu'elle intervienne comme une réactualisation des œuvres du répertoire afin d'en proposer une lecture républicaine, révolutionnaire ou romantique, la mise en scène s'élabore comme un discours historiographique. J. Vilar, dans sa Lettre aux associations populaires de 1961-1962, l'avait clairement dit :

Peu importe que la comédie ou le drame, fidèle miroir de nos difficultés de 1960 ou 1961, soit une œuvre vieille de vingt siècles. Le problème de Cuba est traité dans le Nicomède de Corneille. Le problème du droit des gens devant la loi est traité dans Antigone de Sophocle. Le problème du Général de Gaulle est traité peut-être dans le Cinna de Corneille et dans L'Alcade de Zalaméa.

La mise en scène s'offre donc comme un langage qui s'approprie les problèmes du monde pour en offrir une lecture politisée. Peu importe finalement qu'elle prenne appui sur un texte dramatique, des éléments performatifs ou des matériaux puisés dans le monde "réel», elle se définit essentiellement dans la relation intersubjective entre des artistes et un public, dans sa façon de situer le théâtre comme le lieu adéquat pour recréer le corps social et lui permettre d'avancer dans sa connaissance politique, économique et éthique du réel. Les collectifs actuels délaissent de plus en plus les grandes œuvres du répertoire au profit de nouvelles stratégies d'écriture, disparates dans leurs manières de représenter et d'analyser le réel. Ne satisfont-ils pas pour autant une autre définition de la mise en scène ? P. Pavis (2017, p. 30), à l'issue de ses réflexions sur la mise en scène contemporaine, semble conclure en ce sens :

Le retour de la mise en scène, ou plus exactement sa survie sous d'innombrables nouvelles formes, nous renvoie, au-delà des figures changeantes du metteur en scène, à une conception pragmatique de la mise en scène. Ce qui compte en effet, ce n'est pas une notion figée de ce qu'elle fait ou devrait faire, c'est ce qu'elle nous permet de penser en guidant notre regard : comment elle nous éclaire sur tel ou tel aspect de notre monde, ce en quoi elle nous aide à faire front, ce qu'elle nous aide à penser et à espérer.

Dans cette perspective, la mise en scène se définit bien comme un langage qui articule des morceaux de réel et une production d'illusion de façon à réfléchir le monde.

\section{BIBLIOGRAPHIE}

ALBERTIN, H. (1829). Indications générales pour la mise en scène de Henri III et sa cour, drame historique en cinq actes, en prose, de M. Alexandre Dumas. Paris : Duverger. En ligne : https://gallica.bnf.fr/ ark:/12148/bpt6k57722302.texteImage. 
ALLÉVY, M.-A. (éd.) (1938). Édition critique d’une mise en scène romantique. Indications générales pour la mise en scène de Henri III et sa cour. Paris : Droz

ANToIne, A. (1903). « Causerie sur la mise en scène ». In : Sarrazac, J.-P., \& Marcerou, P. (éds), Antoine, l'invention de la mise en scène. Arles : Éd. Actes Sud, p. 106-120.

BENHAMOU, A.-F. (2012). Les Dramaturgies de plateau. Paris : Éd. Les Solitaires intempestifs.

BIET, C. \& TRIAU, C. (2006). Qu'est-ce que le théâtre ? Paris : Gallimard.

DANAN, J. (2013). Entre théâtre et performance : la question du texte. Arles : Éd. Actes Sud.

FolCO, A. (2013). « La querelle sur les origines de la mise en scène moderne et les enjeux mémoriels autour de la figure d'Antoine ». Revue d'Histoire du Théâtre numérique 1, p. 47-56. En ligne : https://hal.archives-ouvertes.fr/hal-01644800/document.

FRAMERY, N.-É. \& GINGUENÉ, P.-L. (éds) (1971). Encyclopédie méthodique, Musique. 2 vol. Paris : Panckoucke/Vve Agasse.

FRANTZ, P. (1992). « Théâtre et fêtes de la Révolution ». In : De Jomaron, J. (dir.), Le Théâtre en France. Paris : A. Colin, p. 505-532.

FRANTZ, P. (1998). L'Esthétique du tableau dans le théâtre du XVIII siècle. Paris : Presses universiatires de France.

GIANNouli, A. (2010). « Antigone de Sophocle : une première mise en scène ». Revue d'Histoire du Théâtre 248 (4), p. 405-424.

GOUHIER, H. (1989). Le Théâtre et les arts à deux temps. Paris : Flammarion.

HASLÉ, L. (2020). La Direction d'Adolphe Lemoine-Montigny au Théâtre du Gymnase de 1844 à 1880 . Thèse de doctorat en Histoire moderne et contemporaine. Paris : Université Paris sciences et lettres.

LAUGIER, E. (1844). De la Comédie-Française depuis 1830, ou Résumé des événements survenus à ce théâtre depuis cette époque jusqu'en 1844. Paris : Éd. Tresse/ Buyreaux de la Gazette des théâtres.

LAMBERT, A. (1892). «Sur les planches, Études de mise en scène ». Revue d'art dramatique, t. 28, p. 67.

LÉCROART, P., PESLIER, J. \& PIANA, R. (2013). « Des écritures et des plateaux. Points et contrepoints en guise de manifeste ». Skén\&graphie 1, p. 9-17. En ligne : https://journals.openedition.org/ skenegraphie/1021.

MARTIN, R. (2013). « Introduction ». In : Guilbert de Pixerécourt, R.-Ch., Mélodrames, tome I : 1792-1800, Martin, R. (éd.). Paris : Classiques Garnier, p. 11-79.

MARTIN, R. (2014). L'Émergence de la notion de mise en scène dans le paysage théâtral français (1789-1914). Paris : Classiques Garnier.

MARTIN, R. (dir.) (2021). « Le Mélodrame ». Cahiers de l'Association Internationale des Études Françaises 73, p. $13-184$

PARIS, P. (1855). De la mise en scène des mystères et du mystère de la Passion. Cours de littérature du Moyen Âge (Leçon du 7 mai 1855). Paris : Éd. du Collège de France.

PAVIS, P. (2017). « Réflexions sur la mise en scène contemporaine ». In : Pluta, I. (dir.). Metteur en scène aujourd'hui. Identité artistique en question ?. Rennes : Presses universitaires de Rennes, p. 13-30. 
POIRSON, M. (dir.) (2008). Théâtre sous la Révolution. Politique du répertoire (1789-1799). Paris :

Desjonquères, p. 11-61.

PY, O. (2005). « Avignon se débat entre les images et les mots ». Le Monde. 29 juill. En ligne :

https://www.lemonde.fr/idees/article/2005/07/29/avignon-se-debat-entre-les-images-et-lesmots-par-olivier-py_676211_3232.html.

RAZGONNIKOFF, J. (2006a). « Petite histoire de la mise en scène avant André Antoine ». Journal des trois théâtres 18.

RAZGONNIKoff, J. (2006b). « Petite histoire de la mise en scène avant André Antoine ». Journal des trois théâtres 20.

RAZGONNIKOFF, J. (2006c). « Petite histoire de la mise en scène avant André Antoine ». Journal des trois théâtres 21.

ROGER, P. (dir.) (2011). « La mise en scène : mort ou mutation? ». Critique 774.

SANDERS, J. B. (1978). André Antoine directeur à l'Odéon. Paris : Minard.

SARRAZAC, J.-P. (1999). « Introduction ». In : Sarrazac, J.-P., \& Marcerou, P. (éds), Antoine, l'invention de la mise en scène. Arles : Éd. Actes Sud, p. 5-27.

SARRAZAC, J.-P. (2005). « Genèse de la mise en scène moderne, une hypothèse ». Genesis (ManuscritsRecherche-Invention) 26, p. 35-50. En ligne : https://www.persee.fr/doc/

item_1167-5101_2005_num_26_1_1363.

SOLOMÉ, J.-L. (1827). Indications générales et observations pour la mise en scène de Les Trois Quartiers, comédie de MM. Picard et Mazères. Paris : Duverger.

TACKELS, B. (2015). Les Écritures de plateau. État des lieux. Paris : Éd. Les Solitaires intempestifs.

TALON-HUGON, C. (2006). « Avignon 2005. Le Conflit des héritages ». Du théâtre hors-serie 16.

TISSIER, A. (1992). Les Spectacles à Paris pendant la Révolution. Répertoire analytique, chronologique et bibliographique. De la proclamation de la République à la fin de la Convention nationale (21 septembre 1792 - 26 octobre 1795). Genève : Droz.

VACQUERIE, A., MEURICE, P. (1844). Antigone, tragédie de Sophocle. Paris : Furne.

VITEZ, A. (1995). Écrits sur le théâtre, II. La Scène 1954-1975. Paris : P.O.L.

\section{NOTES}

1. Sur la notion de «tableau» dans la théorie diderotienne, voir l'ouvrage de P. Frantz (1998).

2. Le système des privilèges dramatiques, en vigueur sous l'Ancien Régime, fut conçu de manière à protéger les théâtres royaux. Le Théâtre-Français avait seul le privilège de représenter le théâtre dialogué de langue française, l'Opéra-Comique pouvait seul jouer les comédies mêlées d'ariettes, l'Académie Royale de Musique avait le privilège du ballet mythologique et de la tragédie lyrique. Les théâtres de la Foire et des boulevards, cantonnés aux pièces muettes et aux divertissements, ne se privèrent toutefois pas de contourner l'interdiction d'avoir recours aux dialogues et à la musique nouvelle. L'utilisation d'écriteaux, de mélodies suggérant des paroles, de tirades proférées depuis les coulisses constitue autant de procédés innovants, largement pratiqués tout au long 
du XVIII $^{e}$ siècle. La liberté des théâtres, votée à l'Assemblée en 1791, leur permit toutefois d'avoir accès au répertoire des théâtres académiques et de jouer des pièces dialoguées de plus grande envergure.

3. Par exemple, l'Encyclopédie méthodique, Musique, éditée par N.-É. Framery et P.-L. Ginguené en 1791 (vol. 1, p.633), précise à propos du compositeur A. Sacchini : «Naturellement paresseux et livré au plaisir, il se donnait du bon temps jusqu'aux approches du terme fixé pour la mise en scène. Alors il s'enfermait, travaillait jour \& nuit, s'excédait de fatigue, s'achevait aux répétitions. »

4. Les limites de cet article nous conduisent à synthétiser les exemples. On trouvera ces éléments davantage détaillés dans notre ouvrage (Martin, 2014).

5. On pourra, sur ce point, consulter notre "Introduction" aux Mélodrames de Pixerécourt (Martin, 2013) ou encore le dossier thématique consacré au " Mélodrame " dans les Cahiers de l'Association Internationale des Études Françaises (ibid., 2021).

6. Ces livrets de mise en scène furent édités par les deux directeurs de la scène du Théâtre-Français: J.-L. Solomé, (1827) ; Albertin, H. (1829) ; puis réédité par M.-A. Allévy (1938).

7. Le spectacle avait été commandé par le roi de Prusse Frédéric-Guillaume IV. Nouvellement couronné et favorable aux idées romantiques, il nomme F. MendelssohnBartholdy maître de la chapelle du roi. Antigone est la première œuvre qui lui est commandée dans ce cadre. La pièce fut créée au Nouveau Palais de Postdam le 28 octobre 1841, avant d'être redonnée plusieurs fois à Berlin. A. Vacquerie et P. Meurice ont travaillé leur adaptation à partir du manuscrit de la mise en scène allemand, conservé aujourd'hui à la Maison Victor Hugo (sur ce point, Giannouli, 2010, p. 405-424).

8. Elle constitue la « marque » de l'esthétique scénique d'A. Antoine en effet, comme on peut le constater dans les nombreuses photographies de scène, conservées dans le fonds du "Théâtre de l'Odéon » des Archives Nationales (cote 55 AJ 351-359).

9. Remarquons qu'A. Lemoine-Montigny, directeur du Théâtre du Gymnase, procédait de même, sans pour autant constituer un " milieu » savamment réfléchi en amont des répétitions, si l'on en croit l'article d'A. Lambert (1892): «Il voulait assujettir les artistes aux difficultés d'un décor très meublé. Il emplissait toute la scène au hasard: table au milieu, chaises, canapés, causeuses, piano, tabourets, poufs, consoles, etc. À peine restait-il quelque ruelle parmi cet encombrement. Là-dedans, il fallait se dépêtrer, jouer la comédie. Montigny prétendait - avec raison - que dans la vie, il en était ainsi, et qu'on vivait, marchait dans les salons, dans des appartements surchargés de meubles, bibelots, d'accessoires abondants, et qu'on y jouait toutes les scènes possibles en se servant de ces mêmes obstacles. Absolument vrai ! Et il donnait, disait-il ainsi, l'impression exacte de la nature, et un grand effet de vérité à la comédie moderne ». Sur A. Lemoine-Montigny, voir aussi la thèse de L. Haslé (2020). 


\section{RÉSUMÉS}

Cette étude met en perspective les conditions d'émergence de la notion de " mise en scène » dans le paysage théâtral français. Elle montre que la «mise en scène " n'engage pas seulement, en amont des expérimentations naturalistes d'André Antoine, une façon d'organiser les éléments de la représentation. Elle est davantage assimilable à une écriture scénique qui complète le drame, lui accorde une portée sociale, esthétique, politique immédiate. Un détour par les scènes des $\mathrm{XVIII}^{\mathrm{e}}$-XIX ${ }^{\mathrm{e}}$ siècles permet ainsi de contextualiser l'avènement de la "mise en scène moderne ", d'identifier le geste inaugural d'Antoine dans sa façon d'appréhender nouvellement la relation texte/scène, de construire les outils théoriques utiles pour interroger les changements de paradigme à l'œuvre dans les créations scéniques d'aujourd'hui.

This study puts into perspective the conditions of the emergence of $t$ he notion of "mise en scène" in the French theatrical landscape. It shows that the "mise en scène"does not only involve, upstream of André Antoine's naturalistic experiments, a way of organizing the elements of the performance. It is more akin to a scenic writing that complements the drama and gives it an immediate social, aesthetic, and political scope. An analysis of the $18^{\text {th }}$ and $19^{\text {th }}$ century theater thus makes it possible to contextualize the advent of "mise en scène moderne", to identify Antoine's inaugural gesture in his way of newly apprehending the relationship between text and stage, and to construct useful theoretical tools for questioning the paradigm shifts at work in today's stage productions.

\section{INDEX}

Mots-clés : Mise en scène, théâtre français, xixe siècle

Keywords : Mise-en-scene, French theater, 19th century

\section{AUTEUR}

\section{ROXANE MARTIN}

Université de Lorraine, 2L2S, F-57000 Metz, France] 